\title{
A numerical study of fluid-structure coupled effect of abdominal aortic aneurysm
}

\author{
Yingbo Cong ${ }^{\mathrm{a}}$, Liya Wang ${ }^{\mathrm{a}, \mathrm{b},{ }^{*}}$ and Xiao Liu ${ }^{\mathrm{a}}$ \\ ${ }^{a}$ Department of Engineering Mechanics, School of Mechanical Science and Engineering, Jilin \\ University, No. 5988 Renmin Street, Changchun 130022, China \\ ${ }^{b}$ Department of Architectural Engineering, Guangdong Country Garden Polytechnic, No. 2, \\ Daxuedong Road, Qingyuan, 511510, China
}

\begin{abstract}
Three numerical models of an abdominal aortic aneurysm (AAA) with different geometric parameters are established in order to examine the coupled effect of the fluid-structure of AAA. The study is focused on examining the roles of the dilatation parameter and the aspect ratio of an AAA in the flow dynamic within a pulse period. The numerical results demonstrate that the vortex dominates the dynamic flow behavior within an AAA. During a pressure impulse cycle, an AAA is influenced by the entirety of the vortex, from its generation to its subsequent disappearance. As an indirect effect of the vortex dynamic acting on vessels, a series of alternate impulse responses of the wall shear stress (WSS) is generated in an AAA as eddies induced by the vortex move along the axis of the vessels, and the strength of the responses is proportional to the strength of the eddies. The alternated impulse of WSS may be one of the main influencing factors on AAA rupture. The results of this study aided in understanding the mechanisms underlying the evolutionary processes of AAAs.
\end{abstract}

Keywords: Abdominal aortic aneurysm, fluid-structure interaction, numerical analysis, geometric parameters, vortex dynamic, wall shear stress

\section{Introduction}

Abdominal aortic aneurysm (AAA) is a prevalent disease that is characterized by a localized, permanent dilatation of the infrarenal abdominal aorta, similar to a balloon [1]. AAAs pose a health risk due to potential thrombus formation and dissection; however, most importantly, if an AAA is left untreated, the aneurysm will continue to steadily grow until it ruptures [2]. Diagnosis and treatment of this vascular disease have become priorities in the health management of the elderly population. However, due to a lack of knowledge concerning the role that various factors play in the expansion process, there is currently no accurate technique to predict the aneurysmal expansion rate or to determine its critical size (or shape) at the point of rupture [3]. Moreover, an important component of further understanding AAA is a greater understanding of hemodynamic factors, which have been widely accepted to be important to AAA pathogenesis. Furthermore, from a flow mechanics standpoint, it can be anticipated that changes in the geometry of an AAA will result in changes to the

\footnotetext{
* Address for correspondence: Liya Wang, Department of Engineering Mechanics, School of Mechanical Science and Engineering, Jilin University, No. 5988 Renmin Street, Changchun 130022, China. Tel.: 86-0431-85095834; Fax: 86-043185095288; E-mail: wla@jlu.edu.cn.
} 
blood flow patterns within the aneurysm, as well as result in changes to the hemodynamic stresses on its wall. The hemodynamic stresses associated with (or leading to) changes in the aortic wall affects the mechanical integrity and, consequently, results in aneurysm dilation and eventual rupture [4]. Therefore, the identification of specific hemodynamic factors responsible for the growth of the aneurysm may lead to the prevention of AAA.

During the last decade, considerable research has been focused on computational and experimental in vitro studies aimed at providing information on the flow characteristics in either idealized or patient-specific aneurysm shapes [5]. For example, Di Martino, et al. [6] investigated the complex mechanical interaction between the blood flow and wall dynamics in a custom model of AAA by means of computational coupled fluid-structure interaction analyses. In their study, velocity and pressure quantification in addition to quantification of the stresses acting in the AAA wall due to blood flow and arterial wall mechanics were provided. In order to investigate the association of aortic wall weakening with AAA rupture, Khanafer Khalil, et al. [4] analyzed the turbulent flow effect and the wall stress on the aortic aneurysm. Their work demonstrated that the flow structure in a numerical model depends on whether the aneurysm wall is rigid or flexible. Moreover, Sheard Gregory [7] simulated the pulsatile flow through a tube featuring a sinusoidal bulge in order to determine the flow dynamics and wall shear stress conditions encountered under conditions representative of blood flow through a human abdominal aortic aneurysm. The results showed that the flow is dominated by a strong vortex ring that develops in the bulge during the systolic phase of the heartbeat waveform.

The purpose of this paper is to investigate the coupled effect of AAA fluid-structure in a series of symmetrical three-dimensional numerical models under two distinct ideal blood pressure waveforms that were established on the pathological features of AAA, including elastic walls and homogeneous incompressible Newtonian fluid. The influence of geometrical parameters on the variation and distribution in the flow dynamic features was investigated. The study focuses on examining the role of both the dilatation parameter $\beta$, and the aspect ratio $\alpha$ of AAA on the flow dynamic within a pulse period. The results hold potential clinical utility for research and for predicting AAA rupture.

\section{Materials and methods}

\subsection{Geometrical models}

The simulation is composed of a parametric study in axisymmetric models of AAA. The models tested in the numerical study are made of a fusiform expansion and blown in a straight tube, as shown in Figure 1(a). Although the physiologically correct geometry is not preserved, the models still retain all the important physical processes that occur in an AAA.

The geometric parameters of the models follow that of Salsac, et al. [3] and are given by Eqs. (1) and (2):

$$
\begin{array}{ll}
\text { The aspect ratio: } & \alpha=L / 2 R_{0} \\
\text { The dilatation ratio: } & \beta=R / R_{0}
\end{array}
$$

where $R_{0}$ and $L$ are the inner radius of the parent vessel and the length of the aneurysm bulge, respectively, and $\mathrm{R}$ is the maximum inner radius. The total length of the aneurysm is approximately $3 \mathrm{~L}$. For convenience, the AAA model is divided into four segments, as is shown in Figure 1(b). Moreover, 


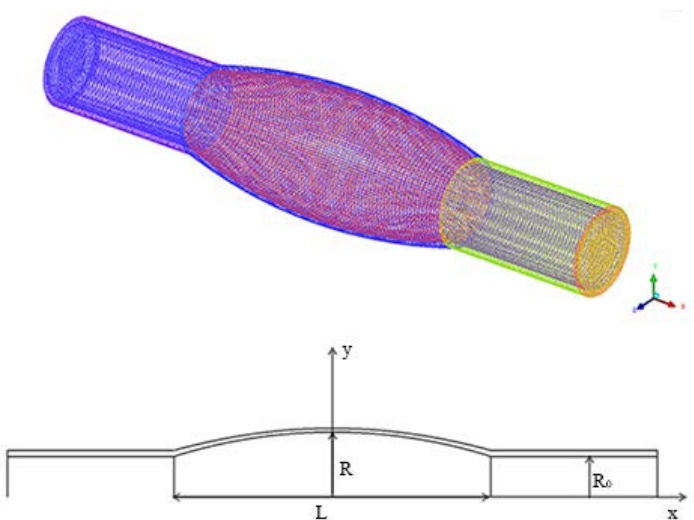

(a)

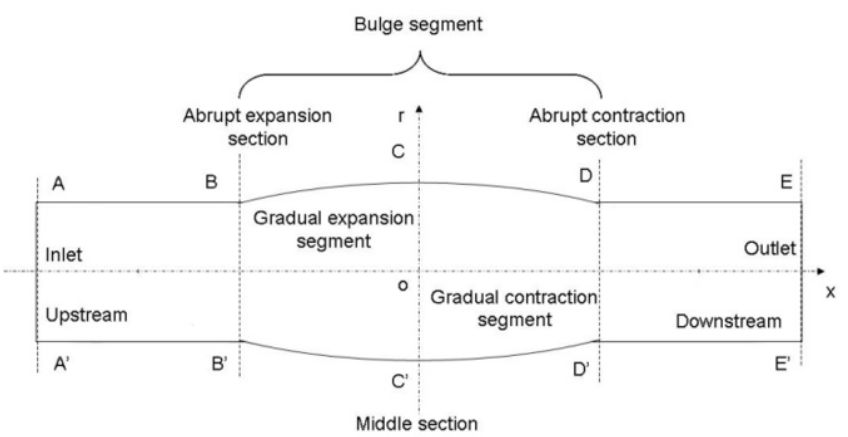

(b)

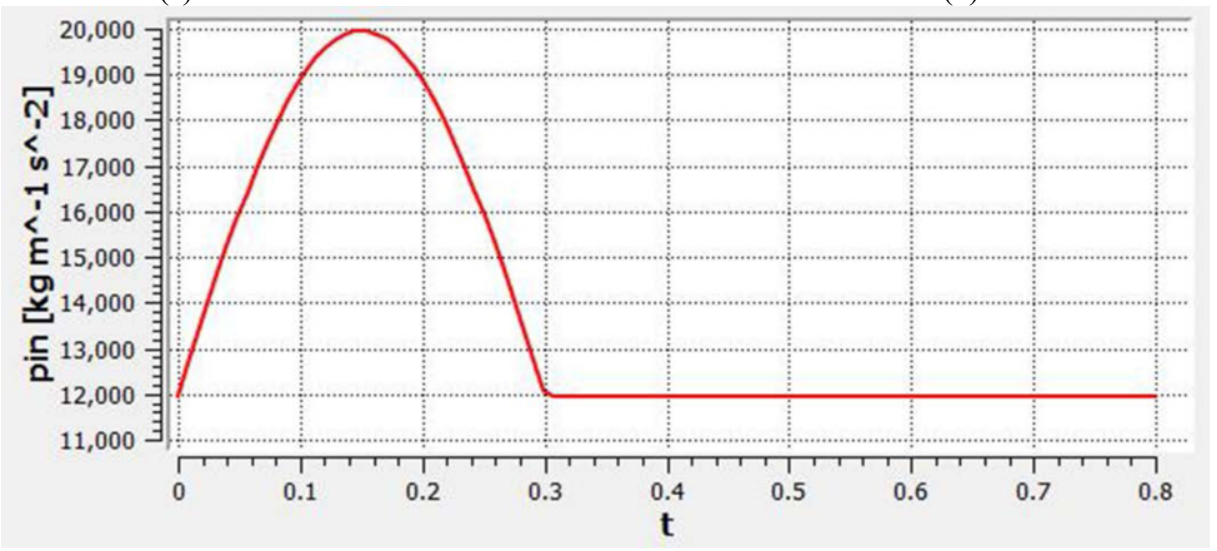

(c)

Fig. 1. (a) 3D meshes and longitudinal profile of Model 3; (b) Schematic drawing for the compliant models; (c) Pressure waveform imposed at the inlet under high pressure case.

$\mathrm{AA}^{\prime}, \mathrm{BB}^{\prime}, \mathrm{CC}^{\prime}, \mathrm{DD}$ ', and EE' respectively denote the inlet section, the abrupt expansion section, the midsection of the bulge, the abrupt contraction section, and the outlet section of the vessels. The $\mathrm{AB}$ segment is located upstream of the BB' section. The gradual expansion segment (BC) is located downstream of the $\mathrm{BB}^{\prime}$ section and upstream of the $\mathrm{CC}^{\prime}$ section. The gradual contraction segment (CD) is located downstream of the CC' section and upstream of the DD' section, and finally, the DE segment is located downstream of the DD' section (Figure 1(b)).

Three virtual aneurysm models were established using different combinations of geometric parameters $\alpha$ and $\beta$. It is advantageous that these well-controlled geometrical shapes are characterized by only two parameters. In this study, these parameters are varied in order to examine changes to the hemodynamic factors as the aneurysm enlarges (see Table 1).

In this study, the grid system was associated with the solid domain and the fluid domain. The

Table 1

Geometric parameter of the models

\begin{tabular}{lccc}
\hline & Model 1 (M1) & Model 2 (M2) & Mode 3 (M3) \\
\hline$\alpha$ & 2.9 & 2.9 & 3.9 \\
$\beta$ & 1.3 & 1.6 & 1.6 \\
\hline
\end{tabular}


computational domain was meshed with eight-node linear hexahedral elements in the software, ICEM/CFD. Figure 1(a) shows the grid of the computational domain, and there is a total of 570,248 cells for the 3D domain.

\subsection{Governing equations}

The governing equations for the fluid domain are the continuity and Navier-Stokes equations, which are shown below:

$$
\begin{gathered}
\text { (Continuity) } u_{i, i}=0 \\
\text { (Momentum) } \rho \frac{\partial u_{i}}{\partial t}+\rho\left(u_{j}-\hat{u}_{j}\right) u_{i, j}=\sigma_{i j, j}+\rho f_{i} \quad \text { in }{ }^{F} \Omega(t)
\end{gathered}
$$

where $u_{i}$ is the velocity, $\rho$ is the fluid density, $\sigma_{i j}$ is the stress, $f_{i}$ is the body force at time t per unit mass, $\hat{u}_{j}$ is the mesh velocity at time $\mathrm{t}$, and ${ }^{F} \Omega(t)$ is the moving spatial domain upon which the fluid is described.

The general governing equations for the structural dynamics are:

$$
\begin{aligned}
& \text { (Momentum) } \rho_{w} \frac{d \hat{u}_{i}}{d t}=\sigma_{i j, j}+\rho_{w} f_{i} \text { in }{ }^{s} \Omega(t) \\
& \text { (Equilibrium of condition) } \sigma_{i j} n_{i}=T_{i} \text { on }{ }^{s} \Gamma(t) \\
& \text { (Constitutive) } \sigma_{i j}=D_{i j k l} \varepsilon_{k l} \text { in }{ }^{s} \Omega(t)
\end{aligned}
$$

Here, ${ }^{s} \Omega(t)$ is the structure domain at time $\mathrm{t}, \rho_{w}$ is the wall density, $n_{i}$ is the outward pointing normal on the wall surface ${ }^{s} \Gamma(t), T_{i}$ is the surface traction vector at time $\mathrm{t},{ }^{s} \Gamma(t)$ is the boundary of the structure domain, $\sigma_{i j}$ is the mechanical stress tensor, $D_{i j k l}$ is the Lagrangian elasticity tensor, and $\varepsilon_{k l}$ is the strain tensor.

Blood was regarded as a homogenous, incompressible, and Newtonian fluid, which is an acceptable assumption for large arteries [8]. Other chosen properties were a dynamic viscosity of $2.7 \mathrm{cP}(0.0027$ $\mathrm{Pa} \mathrm{s}$ ) and a density of $1060 \mathrm{~kg} / \mathrm{m}^{3}$ [6]. Furthermore, the wall material implemented in the model represents an aortic wall tissue of medium characteristics as reported in the Di Martino tests [6]. The wall was supposed to be linear elastic homogeneous; the density was $1120 \mathrm{~kg} / \mathrm{m}^{3}$, the Young modulus was set to $2.7 \mathrm{MPa}$, and the Poisson coefficient was 0.45 .

\subsection{Boundary conditions}

Blood pressure is usually studied under normal conditions, and as such, very few authors have studied high pressure conditions. The pulsatile blood pressure waveform was applied at the inlet boundary, and constant pressure was given at the outlet boundary. There was no slip condition at the inner walls. The cardiac cycle period was $0.8 \mathrm{~s}$, with the peak pressure occurring at $0.15 \mathrm{~s}$. The range of the normal pressure and the high pressure were respectively $70 \sim 120 \mathrm{mmHg}$ and $90 \sim 150 \mathrm{mmHg}$, 

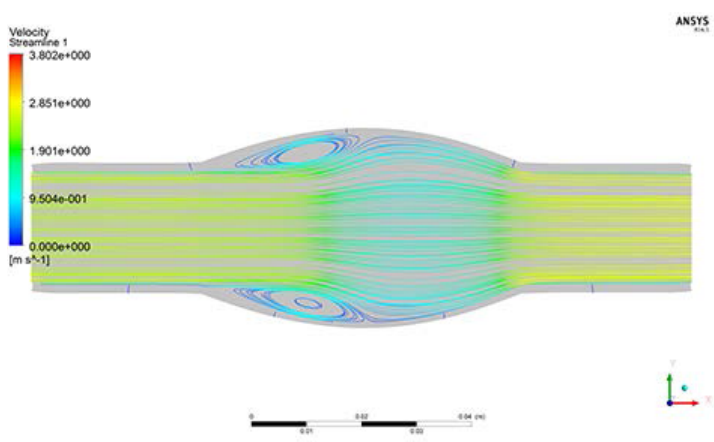

(a)

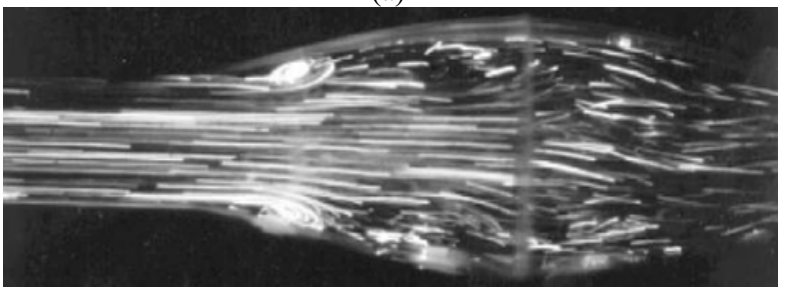

(c)

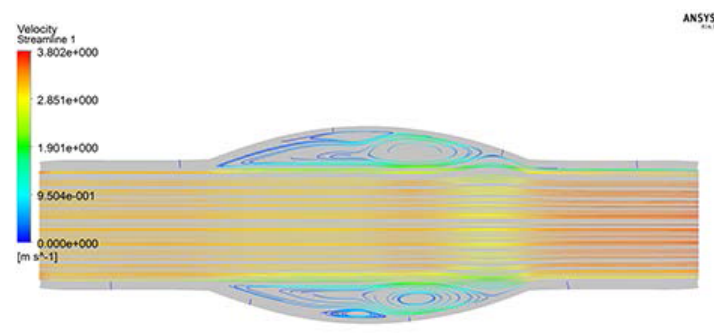

$\stackrel{\leftrightarrow}{\longrightarrow}$ (b)

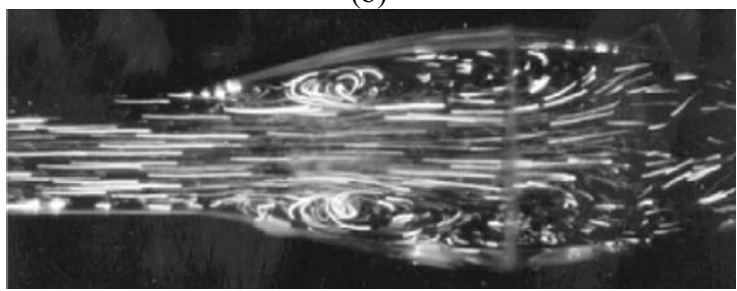

(d)

Fig. 2. Instantaneous streamlines at $\mathrm{t}=0.1 \mathrm{~s}$ (a) and $\mathrm{t}=0.15 \mathrm{~s}(\mathrm{~b})$ in Model 3 under the high pressure case. Vortex formation (c) and vortex translation (d) of the flow visualization photographs for AAA with the exercise waveform [11].

which is depicted in Figure 1(c) $(1 \mathrm{mmHg}=133 \mathrm{~Pa})$. For the structure, the boundary conditions included fixed displacements at the inlet and the exit in addition to free displacement of the outer wall because the peritoneum and the surrounding tissues do not exert any significant pressure on the arterial wall [9]. In order to predict velocity fields and pressure fields at different ideal blood pressure conditions, this study uses a commercial finite element package, ANSYS 14.5, which is a suitable fluid-structure coupled solver that has been used by Z. Li for the last several years [10].

\section{Results and discussions}

\subsection{The distribution characteristic of the streamlines}

Figures 2(a) and 2(b) show instantaneous streamlines at different times on the axis-symmetric plane in the x-direction in Model 3 under the high pressure case. Figures 2(c) and 2(d) respectively show the vortex formation and vortex translation of the flow visualization photographs for AAA with the exercise waveform by Egelhoff [11].

From Figure 2(a), it can be seen that as the pressure pulse wave $(t=0.1 \mathrm{~s})$ accelerates, a pair of axisymmetric eddies develop by separation within the boundary layer of the wall near section BB' of the gradual expansion segment $\mathrm{BC}$ in Model 3. The boundary layer develops from the abrupt expansion section and gradually grows in thickness until it extends to the midsection and forms a wake region. However, at this moment, the flow at the rest region still maintains the basic laminar flow (Figure 2(a)). In fact, these eddies should be induced by an axisymmetric collar vortex-tube around the $x$-axis in the $3 \mathrm{D}$ space. This means that, as the pressure and velocity increase with time, an axisymmetric collar vortex-tube is preliminary generated due to the combined effect of the separation and the shear between the viscous flow and the curved wall of the vessel. The direction of the vortex is 
the same as $\theta$ in the cylindrical coordinate system.

At $t=0.15 \mathrm{~s}$, as the pressure impulse reaches its peak value, the distribution of the streamlines becomes more complicated in Model 3 (Figure 2(b)). The previous pair of eddies induced by the collar vortex tube has moved up to around the contraction segment of the vessel, whereas the new pair of axisymmetric eddies with an opposite rotation subsequently appears behind the former, closer to the vessel wall of the gradual expansion segment (Figure 2(b)). These new eddies are induced by a new collar vortex tube that is subsequently generated due to the further separation induced by the gradual expansion of the wall. This new vortex tube is located behind the previous one and is in the opposite direction. On one side, due to the downstream movement of the eddies, both the separation and the wake region also move further downstream, and the whole space close to the wall of the bulge segment is affected as the pressure pulse reaches its peak. On the other side, the eddies become larger due to the gradually increasing strength of the collar vortex-tube (Figure 2(b)). At this moment, the streamlines are distributed in completely different patterns in the region within the boundary layer of the bulge segment (due to dilatation) and in the rest region. Furthermore, except for the region within the boundary layer of the bulge segment, laminar flow still dominates the dynamical process of flow. Within the boundary layer of the bulge segment, the vortex plays the dominant role in the flow dynamic behavior.

In Egelhoff, et al. (1999), the pulsatile flow in AAA models was experimentally studied by employing flow visualization and laser Doppler velocimetry using physiologically realistic resting and exercise flow conditions. The model in Figures 2(c) and 2(d) had an aspect ratio of $\mathrm{L} / \mathrm{d}=4$ and a dilatation ratio of $\mathrm{R} / \mathrm{R}_{0}=1.8$, which are similar to Model 3. From flow visualization (particle pathlines) in the moderate size AAA model (Figure 2(c)), it was observed that the vortex formed by peak systole at the proximal end of the AAA. During late systole (Figure 2(d)), the vortex translation occurred towards the distal end of the AAA. By comparing Figures 2(a) and 2(c) as well as Figures 2(b) and 2(d), it can be seen that the distribution characteristics of the streamlines in the numerical study correspond to the results of the experiment. Thus, this indirectly verifies that our modeling results are both reasonable and reliable.

\subsection{The temporal variations of the WSS of the vessels}

Figure 3(a) shows the sketch map of the interaction between the vessel wall and the flow along the bulge segment during an input pressure impulse cycle. Figure 3(b) shows the comparison of the temporary variations of the WSS against time for Model 1. Figure 3(c) shows the temporary variations of the WSS acting on the small segment located near the abrupt compaction cross section for the three models.

From Figure 3(a), it is evident that the WSS between the eddy center and its downstream is positive, but the WSS between the eddy center and its upstream is negative because the normal gradient of the pressure is always positive from the eddy center to its downstream or upstream. Therefore, the WSS along the bulge segment should rapidly generate an alternate response to the input pressure impulse due to the effect of the vortex during a pressure impulse cycle. It is obviously different from those in the other segments of the vessel, and it is demonstrated by the temporary variations of the WSS during a pressure impulse cycle (Figure 3(b)).

From the pressure pulse viewpoint, the WSS in the bulge segment generates a series of alternate impulse responses with respect to the pressure impulse wave, as shown in Figure 3(b). The maximum alternate impulse of the WSS of each small segment along the bulge segment occurs in turn from upstream to downstream as the pressure impulse increases. For example, the alternate impulse of the 


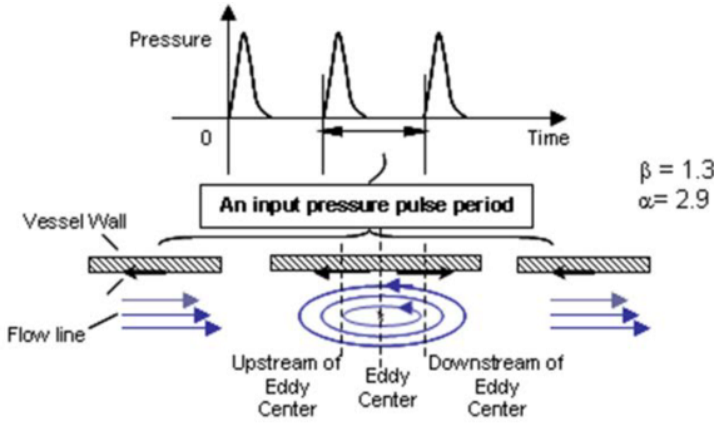

(a)

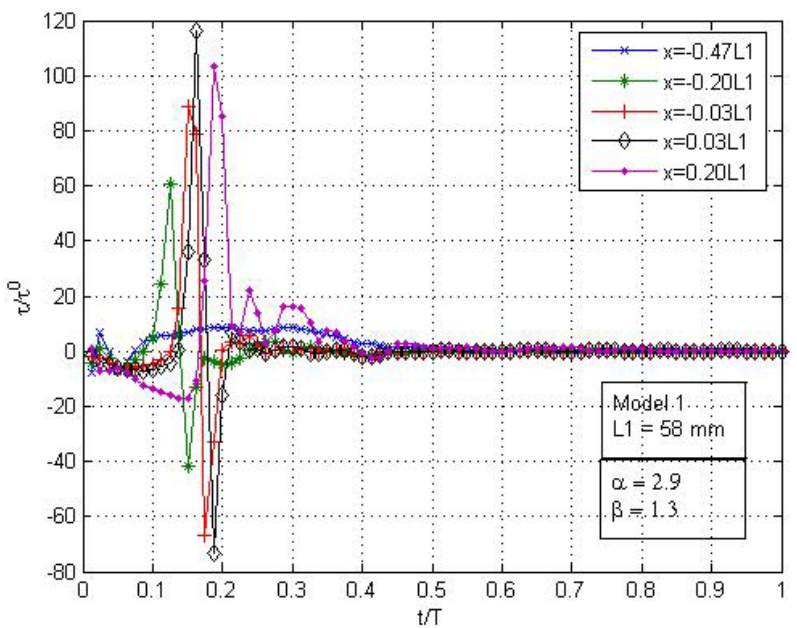

(b)

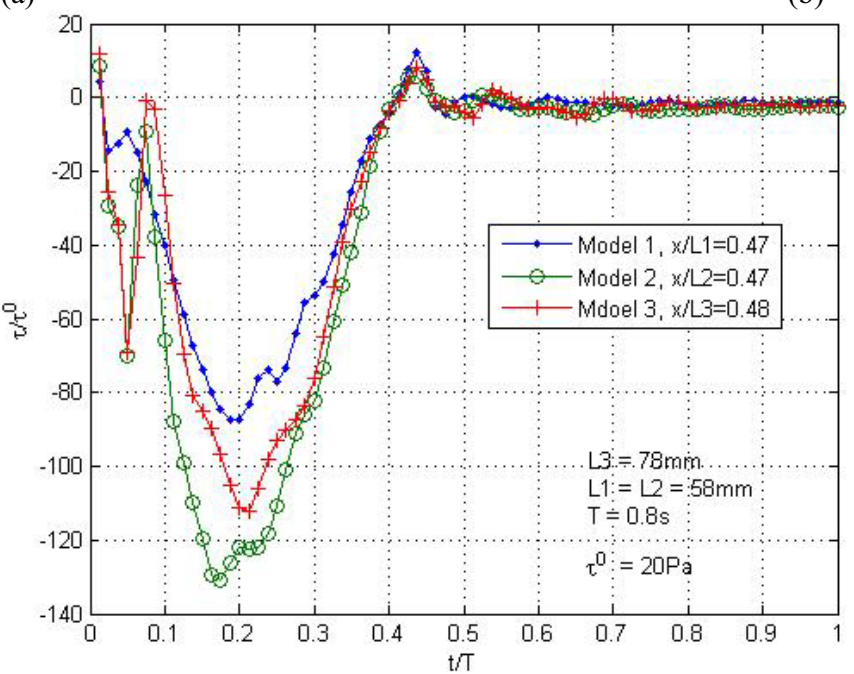

(c)

Fig. 3. (a) The sketch map of the interaction between the vessel wall and the eddy flow; (b) Comparison of the temporal variations of WSS for Model 1 under the high pressure case; (c) Comparison of the WSS at the mini-segment near the abrupt compaction section for the three models.

WSS at the locations of $x=-0.2 \mathrm{~L}$ and $x=-0.03 \mathrm{~L}$ occur at $t=1 / 8 \mathrm{~T}$ and $3 / 20 \mathrm{~T}$, respectively. Moreover, the strength of the alternate impulse of the WSS is in turn increased along the expansion segment, with the maximum alternate impulse of the WSS occurring at $x=0.03 \mathrm{~L}$, where the downstream is very close to the midsection of the bulge segment. Its positive impulse occurs at $t=$ $13 / 80 \mathrm{~T}$, while the pressure impulse is approaching its peak value. The strength of WSS is amplified by about 120 times $\tau^{0}$ (Figure 3(b)), which is the mean value of the WSS of the upstream straight segment within a pressure impulse cycle. Its negative impulse occurs at the next $0.02 \mathrm{~s}$ (i.e., $t=15 / 80$ T), whereas the pressure impulse reaches its maximum value, and the absolute strength of the WSS is amplified by about 80 times $\tau^{0}$. At the same time, a slightly smaller positive impulse (about 100 times $\tau^{0}$ ) of the WSS occurs at $x=0.2 \mathrm{~L}$, which is around $2 / 5$ of the gradual compaction segment. However, the WSS of $x=0.2 \mathrm{~L}$ is negative until approximately $t=0.1675 \mathrm{~T}$, and its maximum absolute is about 
$16.9 \tau^{0}$, which occurs at $t=0.15 \mathrm{~T}$. Immediately after the pressure impulse reaches its maximum, it rapidly reduces to its normal level as time increases from $0.1875 \mathrm{~T}$ to $0.2125 \mathrm{~T}$, and the strength of the WSS of each small segment at the bulge vessels rapidly reduces, except for that in the upstream region near the abrupt compaction section. In the upstream region near the abrupt compaction section, the WSS always remains negative, and the absolute strength is proportional to the pressure impulse (Figure 3(c)); moreover, its maximum strength is about $87.5 \tau^{0}$ at $t=0.2 \mathrm{~T}$, immediately prior to the pressure impulse reaching its maximum value.

On the other hand, from the vortex dynamic viewpoint, the response of the WSS along the bulge segment can be roughly divided into three small zones. Zone (1) is the downstream region near the abrupt expansion cross-section BB', where the vortex is primarily generated by separation, and the WSS response at this zone is small. Skipping Zone (2) for just a moment, Zone (3) is the upstream region near to the abrupt compaction cross-section DD', where the vortex has vanished by viscosity. Finally, Zone (2) is the region between Zones (1) and (3), where the vortex is further developed from gradually increasing to gradually decreasing. At the same time, this zone is the region where the most significant alternate impulse responses of the WSS to the vortex occur. Therefore, the following discussion will focus on the WSS alternate impulse response to the vortex in Zone (2).

Firstly, without a loss of generality, the WSS response of a mini-segment at $x=-0.03 \mathrm{~L}$ near the midsection of Zone (2) is examined, as is shown in Figure 3(b). This response can be classified into two steps. In the first step, the WSS is initially very small as the collar vortex tube is located far away from this mini-segment. However, the WSS of this mini-segment is an alternate impulse response to the vortex when the vortex moves into this region. Then, the WSS rapidly increases to about $90 \tau^{0}$ (Figure 3(b)) when the downstream of the eddy center is approaching and when the mini-segment is located between the eddy center and its downstream. Then, the WSS rapidly reduces to zero when the eddy center reaches this mini-segment. This concludes the first stage of the alternate impulse of the WSS, and at this stage, the WSS response to the vortex exhibits only positive impulse behavior. After the conclusion of the first stage and after WSS reaches zero, it then changes directions, and its strength rapidly increases again. This time, the increase in strength corresponds to when the mini-segment is located between the eddy center and its upstream. Then, as the eddy moves away, the strength of the WSS rapidly reduces, like that seen in the first stage. This concludes the second stage of the alternate impulse of the WSS, in which the WSS only exhibits negative impulse behavior. The maximum

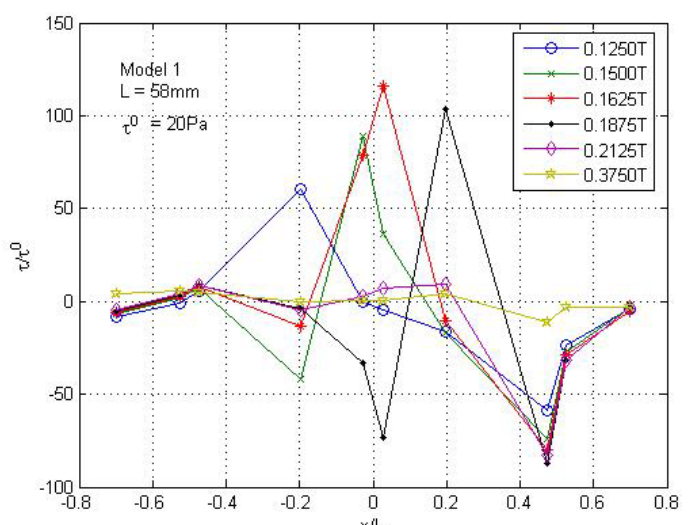

(a)

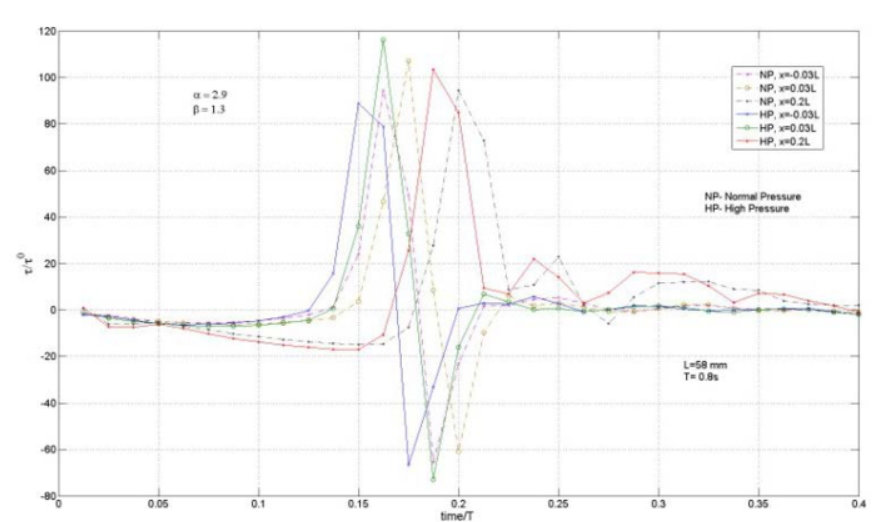

(b)

Fig. 4. (a) Distribution of the WSS along the x-axis-x for Model 1 under the high pressure case; (b) Comparison of the WSS under normal pressure and high pressure for Model 1. 
strength of the negative impulse of the WSS of this mini-segment was approximately $70 \tau^{0}$.

Secondly, the alternate impulse of the WSS along the bulge segment occurs as the eddy center moves along the axis of the bulge segment from upstream to downstream. From among a series of alternate impulses of the WSS along the bulge segment, the largest impulse occurs at the space-time point $\left(x_{\mathrm{m}} \approx 0.03 \mathrm{~L}, t_{\mathrm{m}}=0.175 \mathrm{~T}\right)$ at which the strength of the vortex is at a maximum (Figures $3(\mathrm{~b})$ and 4(a)). The distribution pattern of the WSS along the symmetric axis (Figure 4(a)) also further proves that the most significant alternate impulses of the WSS occur around the midsection of the bulge segment. The cross-section with the largest WSS alternate impulse is located downstream and near the midsection, which is the interface between the gradual expansion and gradual compaction segments. It is obvious that this cross-section is one of the most easily broken down.

By comparing WSS under normal pressure and to that under high pressure (see Figure 4(b)), it can

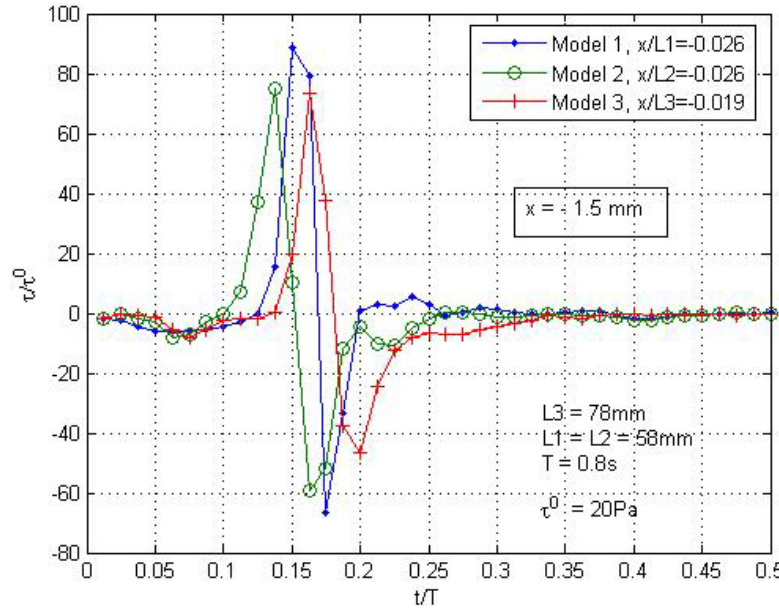

(a)

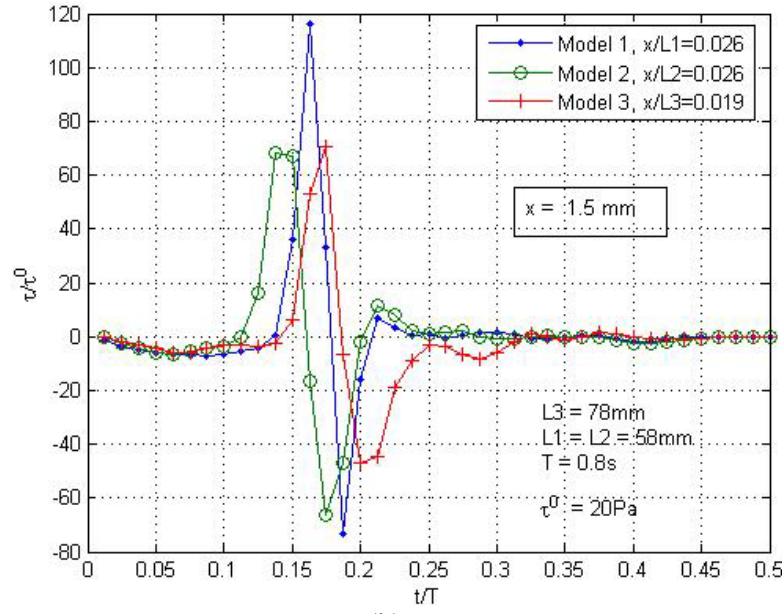

(b)

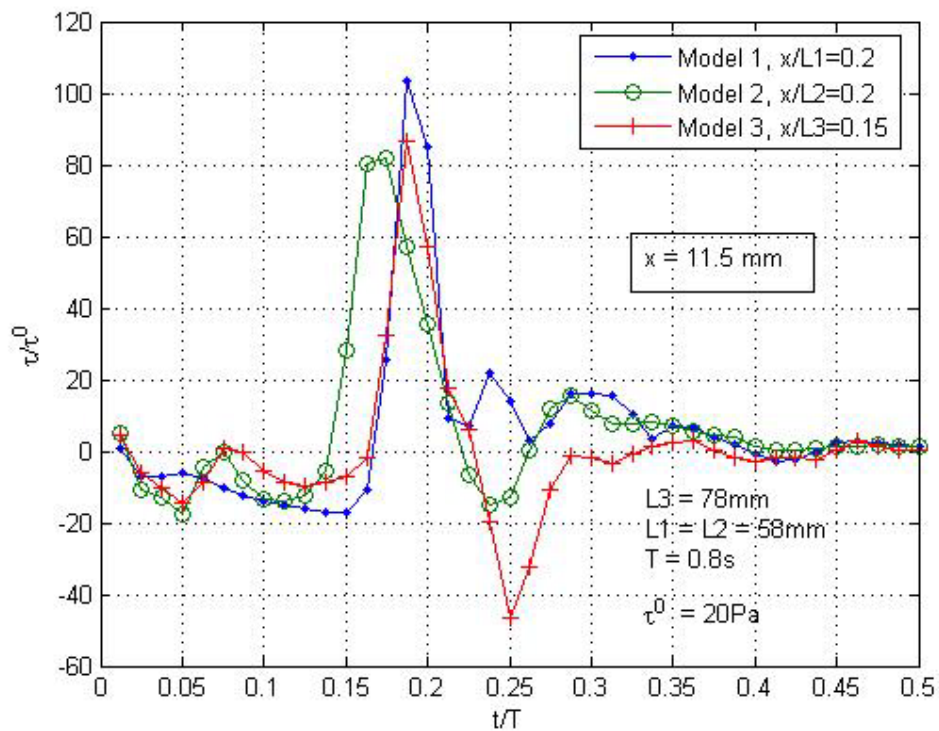

(c)

Fig. 5. Comparison of the WSS for three models under the high pressure case: (a) $x=-1.5 \mathrm{~mm}$, (b) $\mathrm{x}=1.5 \mathrm{~mm}$, and (c) $\mathrm{x}=$ $11.5 \mathrm{~mm}$. 
be determined that: 1) the alternate impulse of the WSS also exists at the bulge segment under the normal pressure case; 2) the maximum WSS alternate impulse is greater and occurs earlier under high pressure than under normal pressure; and 3) the WSS near the mid-section of the bulge segment is very significant, and its maximum is amplified by about 110 times $\tau^{0}$, even if under normal pressure.

\subsection{Effect of the dilation ratio and the aspect ratio on the WSS}

In general, the effects of the geometric parameters on the WSS of the aneurysm are significant, as demonstrated in Figures 3(c) and 5. (Figure 3(c) shows the comparison of the temporal variations of the WSS at the mini-segment near the abrupt compaction section, and Figure 5 shows the comparison of the WSS of the bulge segment from three models during the first 1/2 period of the pressure impulse cycle.) From Figure 3(c), it can be seen that: (1) the absolute maximum of the WSS increases as the dilation ratio parameter $\beta$ increases (demonstrated by comparing the results of Model 1 and Model 2 under the same aspect ratio condition); (2) However, given the same dilation ratio, the absolute maximum of the WSS decreases as the aspect ratio $\alpha$ increases (demonstrated by comparing the results of Model 2 and Model 3); and (3) However, the absolute maximum of the WSS near the compaction section is more sensitive for $\beta$ than for $\alpha$ (demonstrated by comparing the results of Model 1 and Model 3, in which both parameters are increased).

The alternate impulse of the WSS that occurs at the bulge segment is affected by the geometric parameters $\alpha$ and $\beta$ (Figure 5). It should be noted that: (1) the peak value of the WSS decreases when either $\alpha$ or $\beta$ increases (Figure 5(b)); (2) the peak value of the WSS occurs earlier with an increasing dilation ratio $\beta$ (from comparing Model 1 and Model 2 (Figures 5(a)-5(c)), and in contrast, the peak value occurs later with an increasing aspect ratio $\alpha$ (from the comparison of the Model 2 and Model 3 (Figures 5(a) and 5(b)).

\section{Conclusions}

Understanding the role of fluid dynamics in AAA, including the velocity, vortex, shear stress, and pressure evolution, can lead to improved medical care and rupture diagnosis since the internal mechanical forces are maintained by the dynamic action of the blood flowing in the AAAs [1]. This study provides a numerical investigation of the fluid-structure interaction in abdominal aortic aneurysm models as a means of assessing rupture potential non-invasively. It is important to emphasize that the variability in geometrical parameters affects the fluid dynamics in AAAs.

In this study, the flow dynamic process in the AAA was examined by using numerical models. The numerical results demonstrate that the vortex dominated the dynamic behavior of flow within the AAA. During a pressure impulse cycle, an AAA is influenced by the entirety of the vortex, from its generation to its subsequent disappearance. Furthermore, the strength of the vortex largely depends upon the geometric structure of the AAA, and both the dilatation ratio $\beta$ and aspect ratio $\alpha$ play important roles in the entire vortex dynamic. As an indirect effect of the vortex dynamic acting on vessels, a series of alternate impulse responses of the WSS is generated in the AAA as eddies induced by the vortex move along the axis of vessels, and the strength of the responses is proportional to the strength of the eddies. The alternate impulse of WSS may be one of the leading factors of AAA rupture. The absolute maximum of the alternate impulse of WSS increases as the dilation ratio parameter $\beta$ increases, but it decreases with an increasing aspect ratio $\alpha$. The absolute maximum of the 
WSS near the compaction section is more sensitive to $\beta$ than to $\alpha$ when both ratio parameters are increasing.

The above results aided in understanding the mechanisms underlying the evolutionary processes of AAAs. It is significant for its potential research and clinical applicability, particularly in predicting AAA rupture. By employing the fluid-structure interaction methodology, this approach improves the ability to predict AAA biomechanics; however, the present study is limited in some aspects, and these limitations restrict its current applicability to the clinical environment. For example, there remains a need for more efficient models that can incorporate the influence of numerous other factors, such as the realistic geometric shape of an AAA (e.g., profile, tortuosity, and asymmetry), the characteristics of the wall material, the shape and characteristics of the intraluminal thrombus, and so on.

\section{References}

[1] V. Deplano, Y. Knapp, E. Bertrand and E. Gaillard, Flow behaviour in an asymmetric compliant experimental model for abdominal aortic aneurysm, Journal of biomechanics 40 (2007), 2406-2413.

[2] D.A. Vorp, Biomechanics of abdominal aortic aneurysm, Journal of Biomechanics 40 (2007), 1887-1902.

[3] A.-V. Salsac, S.R. Sparks, J.-M. Chomaz and J.C. Lasheras, Evolution of the wall shear stresses during the progressive enlargement of symmetric abdominal aortic aneurysms, Journal of Fluid Mechanics 560 (2006), 19-51.

[4] K.M. Khanafer, J.L. Bull and R. Berguer, Fluid-structure interaction of turbulent pulsatile flow within a flexible wall axisymmetric aortic aneurysm model, European Journal of Mechanics-B/Fluids 28 (2009), 88-102.

[5] C. Stamatopoulos, Y. Papaharilaou, D.S. Mathioulakis and A. Katsamouris, Steady and unsteady flow within an axisymmetric tube dilatation, Experimental Thermal and Fluid Science 34 (2010), 915-927.

[6] E.S. Di Martino, G. Guadagni, A. Fumero, G. Ballerini, R. Spirito and P. Biglioli, et al, Fluid-structure interaction within realistic three-dimensional models of the aneurysmatic aorta as a guidance to assess the risk of rupture of the aneurysm, Medical Engineering \& Physics 23 (2001), 647-655.

[7] G.J. Sheard, Flow dynamics and wall shear-stress variation in a fusiform aneurysm, Journal of Engineering Mathematics 64 (2009), 379-390.

[8] K. Perktold, M. Resch and H. Florian, Pulsatile non-Newtonian flow characteristics in a three-dimensional human carotid bifurcation model, Journal of Biomechanical Engineering 113 (1991), 464-475.

[9] C.M. Scotti, A.D. Shkolnik, S.C. Muluk and E.A. Finol, Fluid-structure interaction in abdominal aortic aneurysms: Effects of asymmetry and wall thickness, BioMedical Engineering OnLine 4 (2005), 64.

[10] Z. Li and C. Kleinstreuer, Fluid-structure interaction effects on sac-blood pressure and wall stress in a stented aneurysm, Journal of Biomechanical Engineering-Transactions of the Asme 127 (2005), 662-671.

[11] C.J. Egelhoff, R.S. Budwig, D.F. Elger, T.A. Khraishi and K.H. Johansen, Model studies of the flow in abdominal aortic aneurysms during resting and exercise conditions, Journal of Biomechanics 32 (1999), 1319-1329. 